\title{
Emergence of noncollinear anisotropies from interfacial magnetic frustration in exchange-bias systems
}

\author{
E. Jiménez, ${ }^{1}$ J. Camarero,,${ }^{1,2, *}$ J. Sort ${ }^{3}$ J. Nogués, ${ }^{4}$ N. Mikuszeit,,${ }^{1,}$ J. M. García-Martín, ${ }^{5}$ A. Hoffmann, ${ }^{6}$ B. Dieny, ${ }^{7}$ and \\ R. Miranda ${ }^{1,2}$ \\ ${ }^{1}$ Departamento de Física de la Materia Condensada and Instituto "Nicolás Cabrera," Universidad Autónoma de Madrid, \\ 28049 Madrid, Spain \\ ${ }^{2}$ Instituto Madrileño de Estudios Avanzados en Nanociencia IMDEA-Nanociencia, Campus Universidad Autónoma de Madrid, \\ 28049 Madrid, Spain \\ ${ }^{3}$ Institució Catalana de Recerca i Estudis Avançats (ICREA) and Department de Física, Universitat Autònoma de Barcelona, \\ 08193 Bellaterra, Spain \\ ${ }^{4}$ Institució Catalana de Recerca i Estudis Avançats (ICREA) and Centre d'Investigació en Nanociència i Nanotecnologia (ICN-CSIC), \\ Edifici CM7, Campus Universitat Autònoma de Barcelona, 08193 Bellaterra, Spain \\ ${ }^{5}$ Instituto de Microelectrónica de Madrid (IMM/CNM-CSIC), 28760 Tres Cantos, Spain \\ ${ }^{6}$ Materials Science Division and Center for Nanoscale Materials, Argonne National Laboratory, Argonne, Illinois 60439, USA \\ ${ }^{7}$ SPINTEC, UMR 8191 CEA/CNRS/UJF, CEA/Grenoble, INAC, 38054 Grenoble Cedex 9, France \\ (Received 26 February 2009; revised manuscript received 17 April 2009; published 15 July 2009)
}

\begin{abstract}
Exchange bias, referred to the interaction between a ferromagnet (FM) and an antiferromagnet (AFM), is a fundamental interfacial magnetic phenomenon, which is key to current and future applications. The effect was discovered half a century ago, and it is well established that the spin structures at the FM/AFM interface play an essential role. However, currently, ad hoc phenomenological anisotropies are often postulated without microscopic justification or sufficient experimental evidence to address magnetization-reversal behavior in exchange-bias systems. We advance toward a detailed microscopic understanding of the magnetic anisotropies in exchange-bias FM/AFM systems by showing that symmetry-breaking anisotropies leave a distinct fingerprint in the asymmetry of the magnetization reversal and we demonstrate how these emerging anisotropies are correlated with the intrinsic anisotropy. Angular and vectorial resolved Kerr hysteresis loops from FM/AFM bilayers with varying degree of ferromagnetic anisotropy reveal a noncollinear anisotropy, which becomes important for ferromagnets with vanishing intrinsic anisotropy. Numerical simulations show that this anisotropy naturally arises from the inevitable spin frustration at an atomically rough FM/AFM interface. As a consequence, we show in detail how the differences observed for different materials during magnetization reversal can be understood in general terms as originating from the interplay between interfacial frustration and intrinsic anisotropies. This understanding will certainly open additional avenues to tailor future advanced magnetic materials.
\end{abstract}

DOI: 10.1103/PhysRevB.80.014415

PACS number(s): 75.70.Cn, 75.50.Ee, 75.60.Jk, 75.25.+z

\section{INTRODUCTION}

The spin arrangement at the interface in layered magnetic materials is often crucial for the understanding of their magnetic properties and has profound consequences for practical applications. Particularly important is the unidirectional coupling between the spins in an antiferromagnet (AFM) and those in an adjacent ferromagnet (FM), referred to as exchange bias. ${ }^{1}$ This effect is widely used to pin the magnetization of thin FM films in today's magnetic applications, ${ }^{2}$ such as magnetic recording, sensors and actuators, and spintronics. Future advances in fields such as ultrahigh-density recording and medical applications are also promoted by effectively increasing the magnetic stability of nanomagnets. ${ }^{3,4}$ A comprehensive description of the effect involves fundamental questions of surface and interface magnetism. ${ }^{5-9}$ The most striking feature is the shift of the FM hysteresis loop along the magnetic field axis, which is widely used in today's spintronic devices to pin the magnetization direction of a FM reference layer. ${ }^{2}$ Moreover, engineering fully adjustable magnetic hysteresis, ${ }^{10}$ as well as the use of nanostructures ${ }^{11}$ or multifunctional materials, ${ }^{12}$ have been recently demon- strated in exchange-coupled FM/AFM systems. In addition, there are a plethora of other magnetic phenomena associated in exchange-coupled FM/AFM systems, such as coercivity enhancements, ${ }^{13,14}$ magnetization reorientation, ${ }^{15-17}$ modified antiferromagnetic spin structures, ${ }^{16,18,19}$ and asymmetric magnetization reversal, ${ }^{20,21}$ which are not fully understood, ${ }^{2}$ and often manifest themselves very differently for various material combinations. Prospects for control, tailor, and enhancement of desirable effects depend upon a clear understanding of the mechanisms governing exchange bias.

The basic underlying physics of exchange bias was already described in the seminal work of Meiklejohn and Bean $^{1} 50$ years ago. Upon cooling a FM/AFM system in an applied magnetic field to below the Néel temperature of the AFM layer, uncompensated spins at the AFM surface align with the polarized FM interface. The often rather small size of the exchange bias has been explained by recent experiments, which show that, unlike for a perfectly flat interface, only a small fraction of uncompensated AFM spins contribute in real FM/AFM systems (which have unavoidable rough interfaces). ${ }^{7,14}$ However, a simple comprehensive understanding of the magnetization reversal of exchange-coupled 


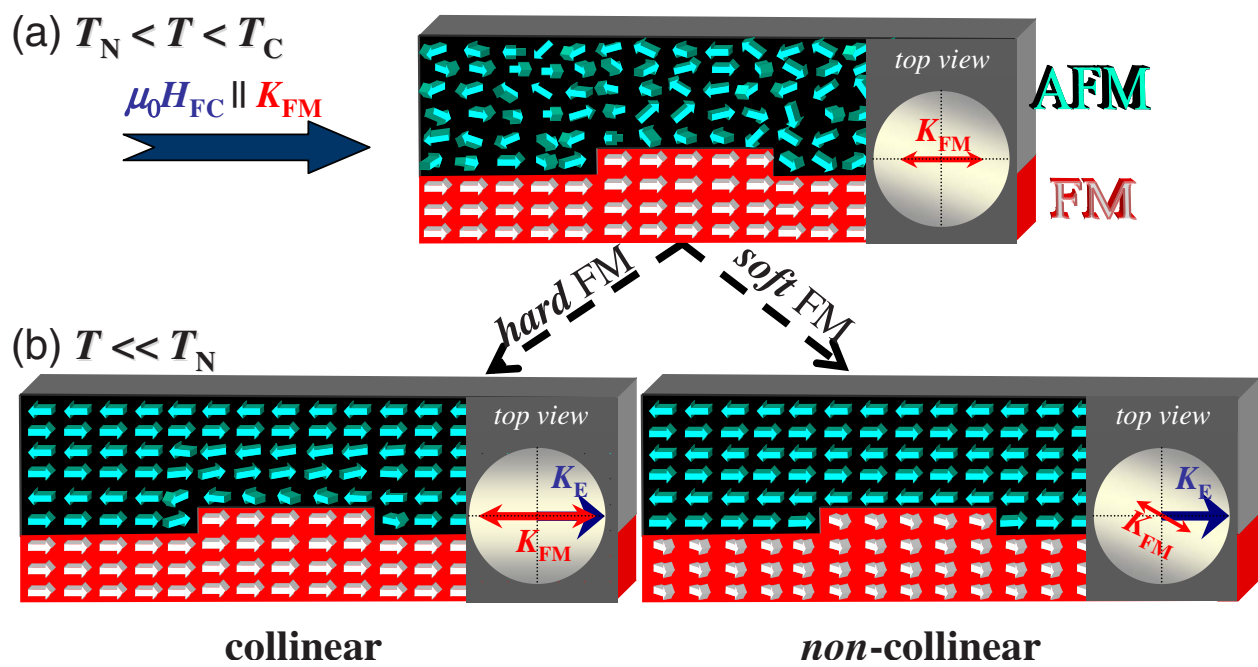

FIG. 1. (Color online) Schematic representation of induced exchange bias in FM/AFM systems: collinear vs noncollinear anisotropy. (a) A ferromagnetic film with well-defined uniaxial anisotropy $K_{\mathrm{FM}}$ in contact with an antiferromagnetic layer is cooled down from above its AFM ordering temperature $\left(T_{\mathrm{N}}\right)$ with an applied external field $\mu_{0} H_{\mathrm{FC}}$ aligned parallel to the FM anisotropy axis. (b) Well below $T_{\mathrm{N}}$, the strength of $K_{\mathrm{FM}}$, with respect to the interfacial unidirectional anisotropy $K_{\mathrm{E}}$ and the frustration at the interface, determines both spin and effective anisotropy (insets) configurations of the FM/AFM bilayer. Note that the spin reorientation of the soft FM layer originates from the coupling frustration at a nonperfect flat FM/AFM interface.

AFM/FM bilayers still remains an unresolved issue. For instance, its associated asymmetric reversal behavior remains one of the poorly understood characteristic features of exchange-biased systems. The different behaviors observed have led to an increasing number of ad hoc magnetic anisotropies being postulated without sufficient microscopic justification.

Here, we show that the angular dependence of the magnetization reversal contains a unique fingerprint of the various effective anisotropies of exchange-bias systems. We used two different FM materials with well-defined uniaxial anisotropy of different strengths, i.e., FeNi and Co, exchange coupled to IrMn to study the influence of the uniaxial, $K_{\mathrm{U}}$, and unidirectional, $K_{\mathrm{E}}$, anisotropies on the magnetization reversal in these bilayers. The samples were field cooled simultaneously with the external field aligned parallel to the easy axis of the FM layer to render samples with collinear anisotropies, i.e., parallel $K_{\mathrm{U}}$ and $K_{\mathrm{E}}$. However, a complete analysis performed in the exchange-bias systems with different ferromagnets reveals that in bilayers with small FM anisotropy a reorientation of the effective anisotropy occurs due to a new noncollinear anisotropy term. A similar noncollinear configuration has already been used to model recent experimental observations in exchange-bias systems. ${ }^{22-25}$ In our case, this is unambiguously deduced from the angular dependence of in-plane angle-resolved hysteresis loops, their asymmetry, and a fit to a modified Stoner-Wohlfarth (SW) model. Additional numerical simulations show that the new anisotropy naturally arises from the inevitable spin frustration at the atomically rough FM/AFM interface, which becomes important for vanishing FM anisotropy. This mechanism of symmetry breaking by interfacial spin frustration confirms the need to use noncollinear anisotropy configurations, as was previously suggested. ${ }^{23,25}$ In addition, our results give further insight into the microscopic mechanism due to the distinctively different behavior for systems with varying intrinsic anisotropy. Our findings hence provide a simple and general explanation, schematically depicted in Fig. 1, on the basis of the ratio of magnetic anisotropies of FM and AFM materials and a new, noncollinear anisotropy contribution, which originates from the frustrated interactions at $\mathrm{FM} / \mathrm{AFM}$ interfaces.

The paper is organized as follows. We describe the experimental details in Sec. II. The results and the discussions are presented in Sec. III. We address the question of the different magnetization-reversal behaviors observed in exchange-bias FM/AFM systems, as originating from the interplay between interfacial frustration and intrinsic anisotropies. A detailed description of the numerical simulations is further reported in the Appendix. The summary is presented in Sec. IV, pointing out that our understanding on the underlying mechanisms of exchange-bias systems will promote new avenues to tailor future magnetic devices.

\section{EXPERIMENTAL}

Reference FM layers and FM/AFM bilayers were sputtered at room temperature (RT) on thermally oxidized Si substrates. The thicknesses of the FM, Co and FeNi, and AFM, IrMn, layers were 12 and $5 \mathrm{~nm}$, respectively. A buffer layer of $5 \mathrm{~nm}$ Ta was employed to favor [111] texture. The Ta layer was deposited at oblique incidence to promote a uniaxial anisotropy in the FM layer. With this method the easy axis of magnetization of the FM layers is in the direction perpendicular to the plane of incidence of the sputtered Ta buffer layer. Finally, the samples were capped by $2 \mathrm{~nm}$ of Ta to prevent oxidation. In order to set the unidirectional anisotropy direction, the samples were annealed at $420 \mathrm{~K}$ for 30 min and field cooled to RT in a $0.25 \mathrm{~T}$ external field aligned in the direction of the FM anisotropy. Note that this procedure should, a priori, render samples with collinear uniaxial 
(a)

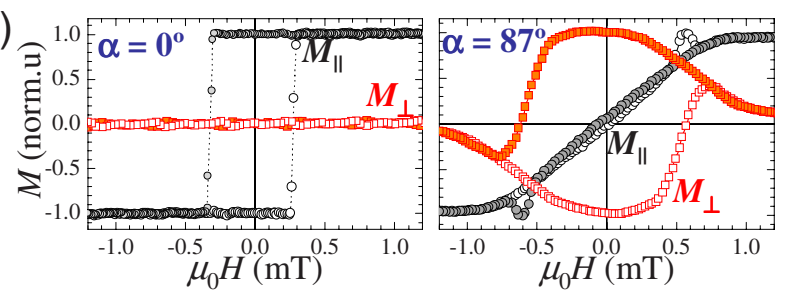

(b)

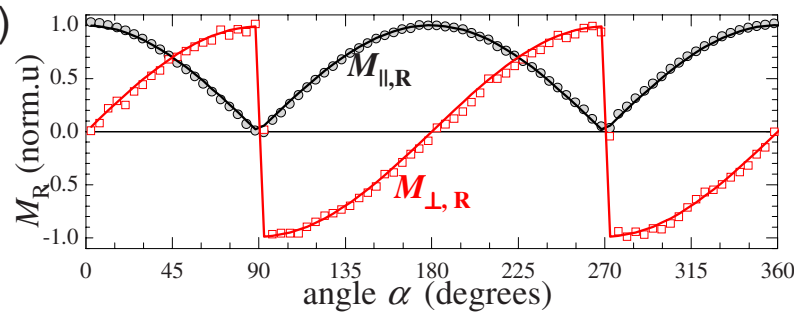

FIG. 2. (Color online) Magnetization reversal of a FM reference sample with well-defined uniaxial anisotropy. (a) Kerr magnetization $M_{\|}(H)$ (circles) and $M_{\perp}(H)$ (squares) loops of a reference 12 $\mathrm{nm}$ FeNi film with the magnetic field applied parallel to the magnetization easy axis (left graph) and close to the magnetization hard axis (right graph). (b) Angular dependence of the remanent $M_{\|, \mathrm{R}}$ (black circles) and $M_{\perp, \mathrm{R}}$ (red squares) as a function of the angle, $\alpha$, with respect to the easy axis at which the magnetic field is applied. The continuous lines correspond to the theoretical curves obtained with the Stoner-Wohlfarth model with uniaxial anisotropy.

and unidirectional anisotropies, i.e., parallel $K_{\mathrm{U}}$ and $K_{\mathrm{E}}$.

The magnetic characterization was performed by highresolution vectorial Kerr magnetometry measurements. In our setup the combination of $p$-polarized incident light in Kerr experiments and the simultaneous detection of the two orthogonal components of the reflected light allow the simultaneous determination of the components of the in-plane magnetization, parallel $\left(M_{\|}\right)$and perpendicular $\left(M_{\perp}\right)$ to the field direction. ${ }^{20} M_{\|}$originates from the difference of the two components of the reflected light, i.e., Kerr rotation, whereas $M_{\perp}$ originates from the small variation in their sum, i.e., reflectivity changes.

The samples are mounted in a stepper motorized eucentric goniometer head, which ensures a constant plane of reflection for the whole measurements performed. In magnetooptical measurements this is important to be able to compare the values of the magnetization components measured at different rotation angles and between different samples. The study of the magnetization-reversal processes and magnetic anisotropy of the film was performed at room temperature by measuring the in-plane-resolved $M-H$ hysteresis loops as a function of the in-plane angular rotation $\theta$ the whole angular range every $0.9^{\circ}$, with $0.5^{\circ}$ angular resolution.

\section{RESULTS AND DISCUSSION}

All reference FM samples display a well-defined uniaxial magnetic anisotropy. Figure 2(a) shows selected magnetization hysteresis loops for the $12 \mathrm{~nm} \mathrm{FeNi}$ film. For $\alpha=0^{\circ}$, the loops are representative for an easy-axis behavior, i.e., square shape with a remanence value similar to the saturation one, i.e., $M_{\|}(0)=M_{\mathrm{S}}$, and a sharp irreversible transition at the coercive field $\left(\mu_{0} H_{\mathrm{C}}=0.3 \mathrm{mT}\right)$, whereas $M_{\perp}(H)$ is zero during the reversal process. A typical hard-axis behavior is found perpendicular to the easy axis. For instance, at $\alpha$ $=87^{\circ}$ the $M_{\|}(H)$ loop is nearly linear with $H_{\mathrm{C}} \approx 0 \mathrm{mT}$. At zero field the magnetization points along the easy axis, i.e., $M_{\|}(0) \approx 0$ and $M_{\perp}(0) \approx M_{\mathrm{S}}$. Both $M_{\|}$and $M_{\perp}$ loops show reversible transitions from the saturation field up to the switching field $\left(\mu_{0} H_{\mathrm{S}} \approx \pm 0.7 \mathrm{mT}\right)$, at which an irreversible transition takes place. The angular dependence of the remanence values of both magnetization components can be reproduced with a simple coherent rotation $\mathrm{SW}$ model for uniaxial anisotropy systems, as shown in Fig. 2(b). The same behavior is found for the Co films, except that the critical fields are six-times larger. This indicates that the effective anisotropy of the Co films is about 1 order of magnitude larger than the one of the FeNi films with similar thickness.

The large difference in FM anisotropy manifests itself in the hysteresis loops measured for the different FM/AFM bilayers. Figure 3 shows representative magnetization loops for hard Co and soft FeNi films exchange biased by an IrMn film measured around the field-cooling direction, i.e., easy axis of magnetization. For $\alpha=0^{\circ}$ (central graphs), the magnetization reverses via a sharp irreversible transition, indicating that the reversal is mainly governed by nucleation and propagation of magnetic domains. For the Co/IrMn bilayer, $M_{\perp}=0$ during the whole magnetization loop, which reflects that the magnetic domains are aligned parallel to the external field. On the contrary, a clear hysteresis is observed in $M_{\perp}$ for the $\mathrm{FeNi} / \mathrm{IrMn}$ bilayer, indicating that the domains are not parallel to the easy axis of magnetization. This unambiguously implies the presence of an additional noncollinear anisotropy and thus a reorientation of the effective FM anisotropy.

Magnetization-reversal asymmetry is found in both bilayers systems for $\alpha \neq 0^{\circ}$. Namely, magnetization reversal via reversible rotation processes is more relevant in one branch of the hysteresis loop, which is characterized by larger $M_{\perp}$ values and rounded transitions in $M_{\|}$. However, the angular dependence of this asymmetry depends on the FM material. In the (hard) Co case, the maximum $M_{\perp}$ is found in the descending branch, i.e., when the field sweeps against the bias direction, independent of the field orientation angle (left panels of Fig. 3). Remarkably, for the (soft) FeNi case the maximum $M_{\perp}$ value can be found in either descending or ascending branches of the hysteresis loop, depending on the sign of the applied magnetic field angle with respect to the easy axis. For positive angles ( $\alpha>0^{\circ}$, bottom right panels), it is found in the descending branch, i.e., when the field sweeps against the exchange-bias direction, similar to the $\mathrm{Co} / \mathrm{IrMn}$ system. On the contrary, for negative angles $\left(\alpha<0^{\circ}\right.$, top right panels) the maximum value is observed in the ascending branch, i.e., when the field sweeps along the bias direction.

Figure 4 shows the angular dependence extracted from hysteresis loops of the normalized exchange bias $\left[H_{\mathrm{E}}(\alpha)\right]$, coercivity $\left[H_{\mathrm{C}}(\alpha)\right]$, and asymmetry $[\zeta(\alpha)$, defined as the difference between the maximum values of $M_{\perp}$ of the descending and ascending branches]. The angular range where the asymmetry is observed for the FeNi/IrMn bilayers is smaller than for the Co/IrMn bilayers, although in both cases it co- 

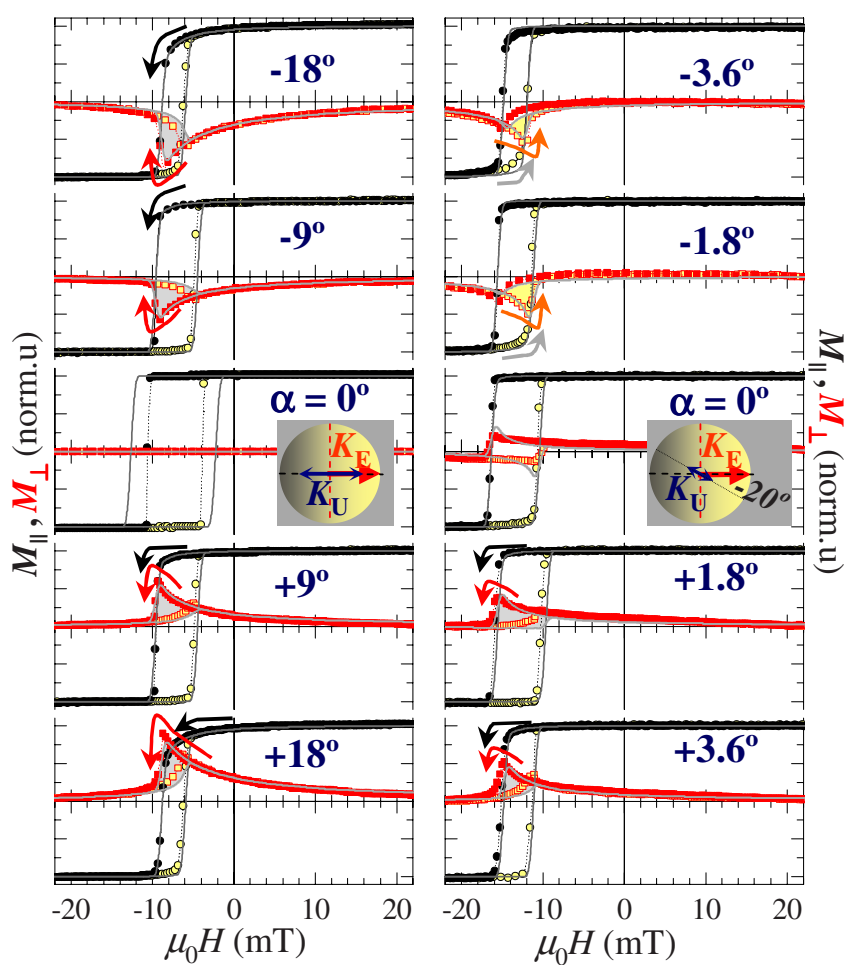

FIG. 3. (Color online) Magnetization-reversal behavior of exchange-coupled FM/AFM bilayers with different intrinsic FM anisotropy. Selected $M_{\|}(H)$ and $M_{\perp}(H)$ hysteresis loops of hard FM (Co, left panels) and soft (FeNi, right panels) films exchange biased to an AFM (IrMn) film for magnetic fields applied at different angles with respect to the easy-axis direction, i.e., field-cooling direction. The two branches of the experimental loops are shown with filled and empty symbols. The continuous lines are the corresponding theoretical curves calculated using a Stoner-Wohlfarth model which includes unidirectional $K_{\mathrm{E}}$ and uniaxial $K_{\mathrm{U}}$ anisotropy constants, as shown schematically in the insets. Different filled area under the $M_{\perp}$ curves and different graph backgrounds have been used to highlight the different asymmetry pathways discussed in the text. Note that the maximum of $M_{\perp}$ is found always in the same branch for Co, whereas it changes for FeNi.

incides with that of irreversible processes. In both systems, the onset of asymmetry $\left(\alpha_{\mathrm{C}}\right)$ coincides with the onset of coercivity and with the maximum of exchange bias and the critical angle $\alpha_{\mathrm{C}}$ increases as the anisotropy ratio $K_{\mathrm{U}} / K_{\mathrm{E}}$ increases. This can easily be understood if the geometrical asteroid solution of the coherent rotation model is used (see Fig. 4 in Ref. 20). In the case of FeNi/IrMn, due to the relatively small intrinsic uniaxial magnetic anisotropy of the FeNi layer compared to the unidirectional anisotropy, the critical angle $\alpha_{\mathrm{C}}$ is smaller. The different asymmetric behavior observed in Fig. 3 is also reflected in $\zeta(\alpha)$. While $\zeta$ changes its sign around the easy axis for the Co/IrMn bilayer, it is always positive for the FeNi/IrMn bilayer.

Calculations based on a coherent rotation SW model including $K_{\mathrm{U}}$ and $K_{\mathrm{E}}$ reproduce our experimental findings, as shown in Figs. 3 and 4 . The parameters $K_{\mathrm{U}}$ and $K_{\mathrm{E}}$ were determined from the experimental data of the reference FM films and the FM/AFM bilayers, respectively (see Appendix). The asymmetric-shape behavior observed for $\mathrm{Co} / \mathrm{IrMn}$
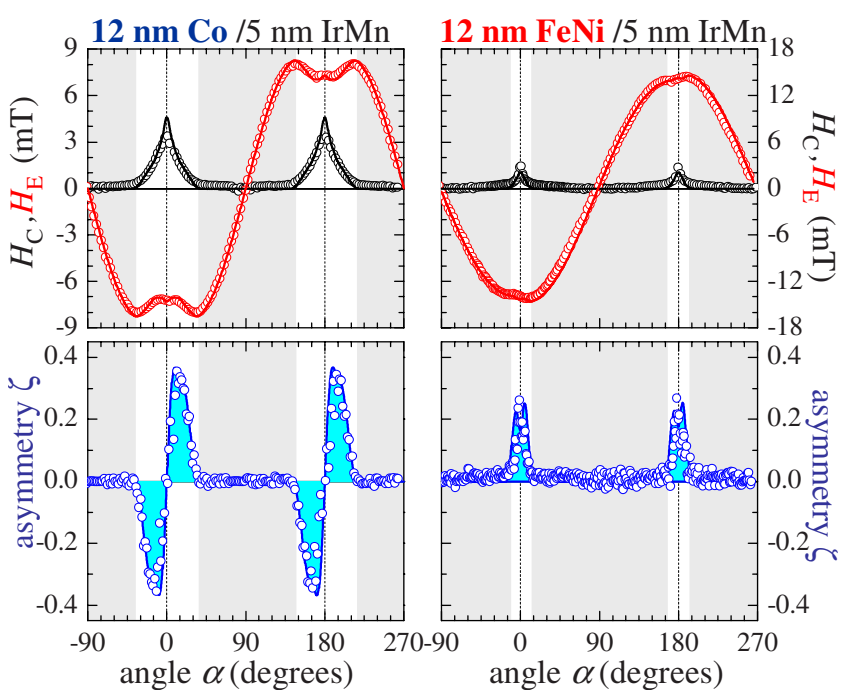

FIG. 4. (Color online) Angular dependence of coercivity, exchange bias, and asymmetry of exchange-coupled bilayers with different intrinsic FM anisotropy. Experimental values (symbols) derived from Kerr measurements such as those shown in Fig. 3, and fit curves (lines) obtained using the SW model including unidirectional $K_{\mathrm{E}}$ and uniaxial $K_{\mathrm{U}}$ anisotropy constants are shown. Collinear and noncollinear $\left(20^{\circ}\right.$ off $)$ anisotropies have been used for the $\mathrm{Co} / \mathrm{IrMn}$ and $\mathrm{FeNi} / \mathrm{IrMn}$ bilayers, respectively. The shadowed areas indicate the range of angles where only reversible processes take place during the reversal. Note that the occurrence of asymmetry is linked with the appearance of irreversibility, i.e., coercivity, and the maximum of the exchange bias. Moreover, the nonsymmetric behavior of the reversal asymmetry found for the FeNi case is reflected by the angular dependence with only positive values.

is well reproduced by considering collinear anisotropies. Surprisingly, although the field cooling was performed with the external field oriented parallel to the easy axis of the original FeNi uniaxial anisotropy, noncollinear anisotropies ${ }^{22-25}$ with a significant misalignment $\left(\theta=-20^{\circ}\right)$ have to be considered to reproduce the hysteresis loops obtained for the FeNi/IrMn bilayers.

These results naturally explain the wide variety of conflicting experimental results for different FM/AFM materials combinations. Asymmetries in the magnetization reversal have been observed for many FM/AFM systems with both in-plane 20-34 $^{20}$ and perpendicular ${ }^{35,36}$ anisotropy for the FM layer. In general, rotation processes are more relevant in one branch of the hysteresis loop, in which a larger density of domains during irreversible domain nucleation processes is also observed. ${ }^{25,28,29,35,36}$ For some systems, rounded transitions in $M_{\|}$and larger $M_{\perp}$ values are found in the descending branch, ${ }^{20,21,25-28,34-36}$ where the field is applied parallel to the exchange-bias direction, while other systems display the opposite behavior. ${ }^{21-25,29-33}$ Our results indicate that this discrepancy is related to the difference between a collinear and a noncollinear anisotropy case. Moreover, the nature of the anisotropy can be unambiguously deduced from the angular dependence of the hysteresis loops.

In order to reach a better understanding of the surprisingly large reorientation of the anisotropy, we performed numerical simulations based on a three-dimensional (3D) cubic lat- 

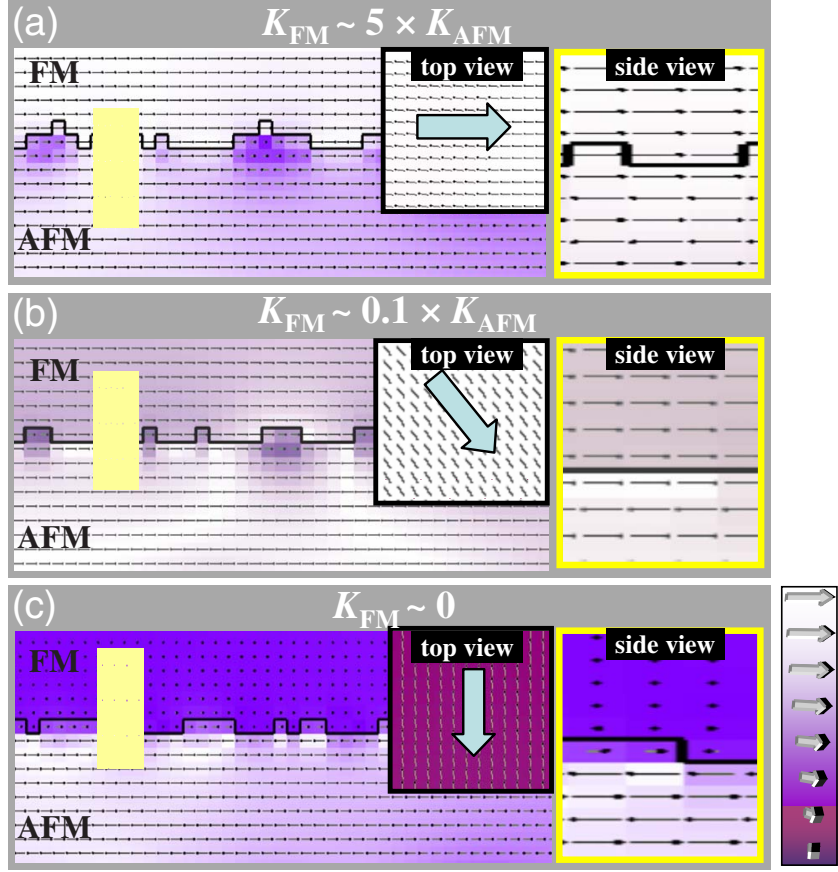

FIG. 5. (Color online) Cut from a 3D simulated spin configuration of FM/AFM bilayers with randomly generated interface roughness. Cross-section views at zero field for different FM anisotropy values, $K_{\mathrm{FM}}$, with a fixed $\mathrm{AFM}$ anisotropy, $K_{\mathrm{AFM}}$ (a) $K_{\mathrm{FM}}=5$ $\times K_{\mathrm{AFM}}$, (b) $K_{\mathrm{FM}}=0.1 \times K_{\mathrm{AFM}}$, and (c) $K_{\mathrm{FM}}=0$. Both AF spin sublattices are shown. The color scale depicted at the bottom right denotes the angle of the spins with respect to the original FM anisotropy easy axis, from 0 (light) to $90^{\circ}$ (dark). The central insets are top views of the spin orientation of the FM layer. The insets at the right show cross-section, side views. Note that the coupling frustration at the rough FM/AFM interface results either in magnetic disorder in the AFM layer (for large FM anisotropy, top panel) or in a spin reorientation of the FM layer (for small FM anisotropy, central and bottom panels).

tice of Heisenberg spins to investigate the spin configuration of the system. Details of the latter are described in the Appendix. A randomly generated roughness was introduced at the FM/AFM interface to induce a magnetic frustration at the interface. Figure 5 shows cross-section views of the magnetic configurations of the system in zero field for different anisotropy values. For $K_{\mathrm{FM}} \geq K_{\mathrm{AFM}}$, a partial spin realignment of the AFM layer at the interface is found [see Fig. 5(a)], consistent with Refs. 25 and 32-34. In the limit of an isotropic FM layer, i.e., $K_{\mathrm{FM}}=0$, the simulation results in an average perpendicular alignment between the FM magnetization and the spin lattice of the AFM layer [Fig. 5(c)], as in a spin flop configuration. ${ }^{37}$ This scenario was observed experimentally for $\mathrm{Fe} / \mathrm{FeF}_{2},{ }^{[38]}$ and $\mathrm{FeNi} / \mathrm{NiO} .{ }^{16}$ For both conditions, the existence of magnetic frustration at the FM/AFM interface implies that the system cannot satisfy all its magnetic interactions simultaneously, which can promote a spin reorientation either in the FM or AFM layer, depending on their anisotropy values. In the same way, for moderate $K_{\mathrm{FM}}$ different partial spin reorientations of the FM layer are obtained depending on the $K_{\mathrm{FM}} / K_{\mathrm{AFM}}$ ratio, as shown in the central insets of Fig. 5. Though the model does not take into account all of the details of real samples, such as the frustration inside the AFM layer (grain boundaries) and chemical intermixing at the interfaces, the results agree qualitatively well. The magnetic frustration, intrinsic to any real sample, and the small $K_{\mathrm{U}}$ of $\mathrm{NiFe}$ can account for the reorientation of the NiFe layer, and thus the concomitant noncollinearity between $K_{\mathrm{U}}$ and $K_{\mathrm{E}}$ observed experimentally. In agreement with the experimental results, no such reorientation effects should take place for large $K_{\mathrm{U}}$.

\section{CONCLUSIONS}

In summary, we have shown that frustration of the exchange coupling between a ferromagnet and an antiferromagnet gives rise to a new noncollinear anisotropy, which becomes important for ferromagnets with vanishing intrinsic anisotropy. The noncollinear nature of the anisotropy is unambiguously deduced from the angular dependence of the hysteresis loops, their asymmetry, and a fit to a modified Stoner-Wohlfarth model. As the intrinsic anisotropy of the ferromagnet is reduced, the exchange coupling to the antiferromagnet results in a reorientation of the effective anisotropy in the ferromagnet. Our findings show that the anisotropy balance and the interfacial magnetic frustration play the main role in the asymmetric reversal phenomena in FM/AFM systems. Our results shed light onto some of the open questions in exchange bias such as reversal asymmetry and especially the observed differences between different materials. This understanding will certainly open new prospects for future applications in magnetic devices.

\section{ACKNOWLEDGMENTS}

J.C. acknowledges support through a "Ramón y Cajal" contract from the Spanish Ministry of Education and Science (MEC). This work was supported in part by the MEC through Projects No. MAT2006-13470, No. MAT200766302-C02, and No. CSD2007-00010 and by the Comunidad de Madrid and the Generalitat de Catalunya through Projects No. S-0505/MAT/0194 (Nanomagnet) and No. 2009-SGR1292, respectively. Work at Argonne was supported by the U.S. DOE-BES through Contract No. DE-AC02-06CH1357.

\section{APPENDIX: NUMERICAL SIMULATIONS}

We have performed numerical simulations to understand the magnetization reversal of the exchange-biased ferromagnetic/antiferromagnetic bilayers as well as the asymmetric phenomena. The experimental results of the angular-dependent magnetization reversal are simulated using a modified Stoner-Wolfhart model in which the anisotropy balance and the angle between them are the basic ingredients (A). The origin of the noncollinearity found for the case of small FM anisotropy is given with a model which combines a three-dimensional lattice of spins and spin disorder (generated by a random interface roughness) to elucidate the spin configuration of the FM/AFM bilayer as a function of the anisotropy balance (B). 
TABLE I. Parameters used to simulate the magnetization loops and the angular dependence of coercivity, exchange bias, and asymmetry. Notice that the uniaxial $K_{\mathrm{U}}$, unidirectional $K_{\mathrm{E}}$, and rotational $K_{\mathrm{R}}$ terms were determined from the experimental magnetization loops (see text) and only the angle $\theta$ between $K_{\mathrm{U}}$ and $K_{\mathrm{E}}$ was adjusted.

\begin{tabular}{lrccr}
\hline \hline & $\begin{array}{c}K_{\mathrm{E}} \\
(\mathrm{mT})\end{array}$ & $K_{\mathrm{U}} / K_{\mathrm{E}}$ & $K_{\mathrm{R}} / K_{\mathrm{E}}$ & $\begin{array}{c}\theta \\
(\mathrm{deg})\end{array}$ \\
\hline Co/IrMn & 7.4 & 0.33 & 0 & 0 \\
NiFe/IrMn & 14.2 & 0.07 & 0.07 & -20 \\
\hline \hline
\end{tabular}

\section{Magnetization reversal}

To gain further insight into the asymmetry phenomena of the magnetization reversal, we performed numerical simulations based on the SW model ${ }^{39}$ including the intrinsic uniaxial anisotropy of the FM layer $K_{\mathrm{U}}$ and the interfacial exchange anisotropy. The latter is divided in two anisotropy contributions to take into account the experimental observations, i.e., exchange bias and coercivity enhancement, which are associated to the two different states of the uncompensated AFM spins during magnetization reversal of the FM layer. First, a unidirectional contribution $K_{\mathrm{E}}$ which accounts for the effects of the pinned AFM spins, responsible for the shift of the hysteresis loop. Second, a rotatable contribution $K_{\mathrm{R}}$ which simulates the effects of the nonpinned AFM spins, responsible of the coercivity enhancement observed in the bilayers. In our systems $K_{\mathrm{R}} \ll K_{\mathrm{E}}$ and it is only necessary to include it, to reproduce the shape of the angular dependence of the coercive field for the FeNi case. The energy per unit volume of the system is, thus, given by

$$
\begin{aligned}
E= & -\mu_{0} M_{\mathrm{S}} H \cos (\alpha)+K_{\mathrm{U}} \sin ^{2}(\alpha-\varphi+\theta) \\
& +K_{\mathrm{E}} \cos (\alpha-\varphi)+K_{\mathrm{R}} \sin ^{2}(\alpha),
\end{aligned}
$$

where $M_{\mathrm{S}}$ is the saturation magnetization, $H$ is the applied magnetic field, and $\varphi$ and $\alpha$ are the angles between the magnetization and the applied field and the field-cooling direction, i.e., easy axis, respectively. The angle $\theta$ is the angle between $K_{\mathrm{U}}$ and $K_{\mathrm{E}}$.

The parameters $K_{\mathrm{U}}, K_{\mathrm{E}}$, and $K_{\mathrm{R}}$ were determined from the Kerr curves (see Table I). The former is extracted from the angular-dependent study of the reference FM layers (i.e., $K_{\mathrm{U}}=\frac{1}{2} H_{\mathrm{K}} M_{\mathrm{S}}$, where $H_{\mathrm{K}}$ is the anisotropy field). $K_{\mathrm{E}}$ is given by the loop shift at $0^{\circ}$ (i.e., $K_{\mathrm{E}}=H_{\mathrm{E}} M_{\mathrm{S}}$ ). $K_{\mathrm{R}}$ was estimated from the comparison between the anisotropy field of the FM layer and the coercive field of the FM/AFM bilayer at $0^{\circ}$, i.e., $K_{\mathrm{R}}=\frac{1}{2}\left[H_{\mathrm{C}}^{\mathrm{FM} / \mathrm{AFM}}\left(0^{\circ}\right)-H_{\mathrm{K}}^{\mathrm{FM}}\right] M_{\mathrm{S}}$. The angle $\theta$ between $K_{\mathrm{U}}$ and $K_{\mathrm{E}}$ was adjusted to simulate the experimental results.
The magnetization loops are extracted via minimization of the total energy of the system (continuous lines in Figs. 3 and 4).

\section{Spin configuration}

The simulations of the spin configuration are based on a three-dimensional lattice of Heisenberg spins, which have been successfully used previously to understand the $90^{\circ}$ coupling observed in FM/AFM/FM systems ${ }^{16}$ as well as the effects of nonmagnetic impurities at FM/AFM interfaces. ${ }^{40}$ The simulations consider a three-dimensional simple cubic lattice with a length of 50 and a width of 32 lattice points. For the thickness, a nominal number of layers of 30 was taken for the FM and 10 for the AFM, with a total of 40 layers. A randomly generated roughness was introduced at the FM/AFM interface to simulate an interface disorder. The roughness in this model consists of randomly distributed discrete monolayer fluctuations and thus neglects interdiffusion effects or more complex interfacial microstructures. The thickness fluctuations contribute to the coexistence of effective positive and negative exchange interactions through the FM/AFM interface, as described in Malozemoff's model, ${ }^{41}$ leading to a strong frustration along this interface.

The total energy $E$ of the bilayer contains three contributions: (i) the Zeeman energy for each layer, (ii) the magnetic anisotropy $K$ of each layer, and (iii) the exchange coupling inside the layers and between the layers at the interface. The energy is thus given by the equation

$$
\begin{aligned}
E= & -\sum_{i, \mathrm{FM}, \mathrm{AFM}}^{\mathrm{N}} \vec{H} \cdot \vec{S}_{i}+K_{\mathrm{FM}} \sum_{i}\left(\vec{n}_{\mathrm{FM}} \cdot \vec{S}_{i, \mathrm{FM}}\right)^{2} \\
& +K_{\mathrm{AFM}} \sum_{i}\left(\vec{n}_{\mathrm{AFM}} \cdot \vec{S}_{i, \mathrm{AFM}}\right)^{2}-J_{\mathrm{FM}} \sum_{i, j>i} \vec{S}_{i, \mathrm{FM}} \cdot \vec{S}_{j, \mathrm{FM}} \\
& -J_{\mathrm{AFM}} \sum_{i, j>i} \vec{S}_{i, \mathrm{AFM}} \cdot \vec{S}_{j, \mathrm{AFM}}-J_{\mathrm{int}} \sum_{i, j>i} \vec{S}_{i, \mathrm{FM}} \cdot \vec{S}_{j, \mathrm{AFM}}
\end{aligned}
$$

A phenomenological coupling was introduced in the proximity magnetism model describing changes in the exchange coupling across the AFM layer with thickness fluctuations. ${ }^{42}$ The exchange coupling constants were estimated from the Néel and Curie temperatures of the AFM and FM bulk materials, respectively. A priori, the same easy magnetization axes direction have been taken for the FM and AFM layers. Different anisotropy constants have been considered to study the effect of the anisotropy balance in the spin configuration of the FM/AFM bilayer. An algorithm based on the conjugated-gradient technique has been employed to minimize the energy of the whole bilayer. ${ }^{16,40,43}$ 
*julio.camarero@uam.es

†Present address: Institut für Angewandte Physik, Universität Hamburg, 20355 Hamburg, Germany.

${ }^{1}$ W. H. Meiklejohn and C. P. Bean, Phys. Rev. 102, 1413 (1956).

${ }^{2}$ See reviews, J. Nogués and I. K. Schuller, J. Magn. Magn. Mater. 192, 203 (1999); A. E. Berkowitz and K. Takano, ibid. 200, 552 (1999); R. L. Stamps, J. Phys. D 33, R247 (2000); M. Kiwi, J. Magn. Magn. Mater. 234, 584 (2001); F. Radu and H. Zabel, in Magnetic Heterostructures: Advances and Perspectives in Spinstructures and Spintransport, edited by H. Zabel and S. D. Bader, Springer Tracts in Modern Physics Vol. 227 (SpringerVerlag, Berlin, 2008), pp. 97-184.

${ }^{3}$ V. Skumryev, S. Stoyanov, Y. Zhang, G. Hadjipanayis, D. Givord, and J. Nogués, Nature (London) 423, 850 (2003).

${ }^{4}$ J. Eisenmenger and I. K. Schuller, Nature Mater. 2, 437 (2003).

${ }^{5}$ F. Nolting, A. Scholl, J. Stöhr, J. W. Seo, J. Fompeyrine, H. Siegwart, J.-P. Locquet, S. Anders, J. Lüning, E. E. Fullerton, M. F. Toney, M.-R. Scheinfeink, and H. A. Padmore, Nature (London) 405, 767 (2000).

${ }^{6}$ H. Ohldag, A. Scholl, F. Nolting, S. Anders, F. U. Hillebrecht, and J. Stöhr, Phys. Rev. Lett. 86, 2878 (2001).

${ }^{7}$ H. Ohldag, A. Scholl, F. Nolting, E. Arenholz, S. Maat, A. T. Young, M. Carey, and J. Stöhr, Phys. Rev. Lett. 91, 017203 (2003).

${ }^{8}$ W. Kuch, L. I. Chelaru, F. Offi, J. Wang, M. Kotsugi, and J. Kirschner, Nature Mater. 5, 128 (2006).

${ }^{9}$ M. Bode, E. Y. Vedmedenko, K. von Bergmann, A. Kubetzka, P. Ferriani, S. Heinze, and R. Wiesendanger, Nature Mater. 5, 477 (2006).

${ }^{10}$ S. Brück, J. Sort, V. Baltz, S. Suriñach, J. S. Muñoz, B. Dieny, M. D. Baró, and J. Nogués, Adv. Mater. 17, 2978 (2005).

${ }^{11}$ J. Nogués, J. Sort, V. Langlais, V. Skumryev, S. Suriñach, J. S. Muñoz, and M. D. Baró, Phys. Rep. 422, 65 (2005).

${ }^{12}$ H. Béa, M. Bibes, F. Ott, B. Dupé, X.-H. Zhu, S. Petit, S. Fusil, C. Deranlot, K. Bouzehouane, and A. Barthélémy, Phys. Rev. Lett. 100, 017204 (2008).

${ }^{13}$ C. Leighton, J. Nogués, B. J. Jönsson-Åkerman, and I. K. Schuller, Phys. Rev. Lett. 84, 3466 (2000).

${ }^{14}$ J. Camarero, Y. Pennec, J. Vogel, S. Pizzini, M. Cartier, F. Fettar, F. Ernult, A. Tagliaferri, N. B. Brookes, and B. Dieny, Phys. Rev. B 67, 020413(R) (2003).

${ }^{15}$ W. Kuch, F. Offi, L. I. Chelaru, M. Kotsugi, K. Fukumoto, and J. Kirschner, Phys. Rev. B 65, 140408(R) (2002).

${ }^{16}$ J. Camarero, Y. Pennec, J. Vogel, M. Bonfim, S. Pizzini, F. Ernult, F. Fettar, F. Garcia, F. Lançon, L. Billard, B. Dieny, A. Tagliaferri, and N. B. Brookes, Phys. Rev. Lett. 91, 027201 (2003).

${ }^{17}$ W. A. A. Macedo, B. Sahoo, V. Kuncser, J. Eisenmenger, I. Felner, J. Nogués, K. Liu, W. Keune, and I. K. Schuller, Phys. Rev. B 70, 224414 (2004).

${ }^{18}$ M. Finazzi, A. Brambilla, P. Biagioni, J. Graf, G.-H. Gweon, A. Scholl, A. Lanzara, and L. Duo, Phys. Rev. Lett. 97, 097202
(2006).

${ }^{19}$ A. Hoffmann, Phys. Rev. Lett. 93, 097203 (2004).

${ }^{20}$ J. Camarero, J. Sort, A. Hoffmann, J. M. García-Martín, B. Dieny, R. Miranda, and J. Nogués, Phys. Rev. Lett. 95, 057204 (2005).

${ }^{21}$ A. Tillmanns, S. Oertker, B. Beschoten, G. Güntherodt, J. Eisenmenger, and I. K. Schuller, Phys. Rev. B 78, 012401 (2008).

${ }^{22}$ H. Xi and R. M. White, J. Appl. Phys. 86, 5169 (1999).

${ }^{23}$ F. Radu, A. Westphalen, K. Theis-Bröhl, and H. Zabel, J. Phys.: Condens. Matter 18, L29 (2006).

${ }^{24}$ M. J. M. Pires, R. B. de Oliveira, Jr., M. D. Martins, J. D. Ardisson, and W. A. A. Macedo, J. Phys. Chem. Solids 68, 2398 (2007).

${ }^{25}$ J. McCord, C. Hamann, R. Schäfer, L. Schultz, and R. Mattheis, Phys. Rev. B 78, 094419 (2008).

${ }^{26}$ V. I. Nikitenko, V. S. Gornakov, A. J. Shapiro, R. D. Shull, Kai Liu, S. M. Zhou, and C. L. Chien, Phys. Rev. Lett. 84, 765 (2000).

${ }^{27}$ M. R. Fitzsimmons, P. Yashar, C. Leighton, I. K. Schuller, J. Nogués, C. F. Majkrzak, and J. A. Dura, Phys. Rev. Lett. 84, 3986 (2000).

${ }^{28}$ A. Kirilyuk, J. Rasing, H. Jaffres, D. Lacour, and F. Nguyen Van Dau, J. Appl. Phys. 91, 7745 (2002).

${ }^{29}$ J. McCord, R. Schäfer, R. Mattheis, and K.-U. Barholz, J. Appl. Phys. 93, 5491 (2003).

${ }^{30}$ P. Blomqvist, K. M. Krishnan, and H. Ohldag, Phys. Rev. Lett. 94, 107203 (2005).

${ }^{31}$ Z.-P. Li, O. Petracic, R. Morales, J. Olamit, X. Batlle, K. Liu, and I. K. Schuller, Phys. Rev. Lett. 96, 217205 (2006).

${ }^{32}$ J. Olamit and Kai Liu, J. Appl. Phys. 101, 09E508 (2007).

${ }^{33}$ S. Brems, K. Temst, and C. Van Haesendonck, Phys. Rev. Lett. 99, 067201 (2007).

${ }^{34}$ T. R. Gao, D. Z. Yang, S. M. Zhou, R. Chantrell, P. Asselin, J. Du, and X. S. Wu, Phys. Rev. Lett. 99, 057201 (2007).

${ }^{35}$ F. Romanens, S. Pizzini, F. Yokaichiya, M. Bonfim, Y. Pennec, J. Camarero, J. Vogel, J. Sort, F. Garcia, B. Rodmaq, and B. Dieny, Phys. Rev. B 72, 134410 (2005).

${ }^{36}$ J. Camarero, J. Miguel, J. Goedkoop, J. Vogel, F. Garcia, F. Romanens, S. Pizzini, N. B. Brookes, J. Sort, and B. Dieny, Appl. Phys. Lett. 89, 232507 (2006).

${ }^{37}$ N. C. Koon, Phys. Rev. Lett. 78, 4865 (1997).

${ }^{38}$ T. J. Moran, J. Nogués, D. Lederman, and I. K. Schuller, Appl. Phys. Lett. 72, 617 (1998).

${ }^{39}$ E. C. Stoner and E. P. Wohlfarth, Philos. Trans. R. Soc. London, Ser. A 240, 599 (1948).

${ }^{40}$ F. Ernult, B. Dieny, F. Lançon, L. Billard, and J. R. Regnard, J. Appl. Phys. 94, 6678 (2003).

${ }^{41}$ A. P. Malozemoff, Phys. Rev. B 35, 3679 (1987).

${ }^{42}$ J. C. Slonczewski, J. Magn. Magn. Mater. 150, 13 (1995).

${ }^{43}$ F. Ernult, Ph.D. thesis, Université Joseph Fourier, Grenoble, 2002. 